\title{
Sudden cardiac arrest in takotsubo cardiomyopathy - a case study
}

\author{
Barbara Sosnowska-Pasiarska¹, Dawid Bąkowski ${ }^{1}$, Agnieszka Woronowicz-Chróściel' \\ Beata Wożakowska-Kapłon ${ }^{1,2}$
}

${ }^{1}$ First Clinical Department of Cardiology and Electrotherapy, Świętokrzyskie Cardiology Center, Kielce, Poland

${ }^{2}$ Faculty of Health Sciences, Jan Kochanowski University, Kielce, Poland

Postep Kardiol Inter 2014; 10, 2 (36): 110-113

DOI: 10.5114/pwki.2014.43517

\begin{abstract}
A bstract
We present a 59-year-old woman who was admitted to hospital after sudden cardiac arrest due to ventricular fibrillation. Finally takotsubo syndrome was diagnosed. In the acute phase of takotsubo syndrome life-threatening ventricular arrhythmias and significant hemodynamic disorders may occur due to strong adrenergic stimulation and myocardial ischemia. It has been proved that the occurrence of torsade de pointes tachycardia in the acute phase of takotsubo cardiomyopathy is associated with QT prolongation. There are no clear guidelines on pharmacological treatment and implantable cardioverter defibrillator implantation after a past takotsubo episode. Takotsubo cardiomyopathy has not been entirely explained as an etiological disease.
\end{abstract}

Key words: takotsubo cardiomyopathy, sudden cardiac death.

\section{Introduction}

ST-elevation myocardial infarction (STEMI) constitutes more than $30 \%$ of all cases of acute coronary syndromes [1]. In the pathogenesis of atherosclerotic plaque, thrombus is the dominant cause of myocardial infarction, arising from the damage site, especially a broken plaque, leading to coronary artery closure, blood flow collapse, and, consequently, to myocardial necrosis. Takotsubo syndrome constitutes about $2 \%$ of all STEMI cases, with most of them occurring in postmenopausal women, after strong emotional or physical stress [2]. The terminology includes numerous names of this syndrome: takotsubo cardiomyopathy, apical ballooning syndrome, transient left ventricular apical ballooning syndrome, transient catecholamine cardiomyopathy, neurogenic myocardial stunning. There are dynamic ST-T segment changes recorded in the electrocardiogram, like in ischemia, and there are some transient contractility disorders shown in echocardiography. Long-term observation in takotsubo syndrome is unburdened in general, but in the acute phase - due to strong adrenergic stimulation and myocardial ischemia - may lead to life-threatening ventricular arrhythmias and significant hemodynamic disorders.

\section{Case report}

A 59-year-old woman was admitted to an intensive cardiac unit (ICU) after sudden cardiac arrest due to ventricular fibrillation, with effective resuscitation having been provided by emergency medical services. Typical chest pain occurred after severe emotional stress caused by a difficult family situation, being the reason for calling the rescue service. Previously, she was treated for diabetes mellitus and chronic obstructive pulmonary disease, with episodes of paroxysmal supraventricular tachycardia also being recorded. At the time of admission to the ICU, the patient was unconscious; the physical examination showed hypotension of $66 / 40 \mathrm{~mm} \mathrm{Hg}$, sinus tachycardia 110 beats per minute, in addition to bronchospastic symptoms having been recorded, with the Glasgow scale assessment of the neurological state scoring 8 . She was treated with mechanical ventilation, catecholamines, bronchodilating medicines, antibiotic therapy and functional insulin therapy. Sinus rhythm, supraventricular extrasystoles and changes of $T$ waves were found in the electrocardiogram (Figure 1). Corrected QT interval calculated with Bazett's formula was 550 ms. Dilated apex and mid-ventricular segment akinesis were recorded in echocardiography. Left ventricular ejection fraction was

\section{Corresponding author:}

Barbara Sosnowska-Pasiarska MD, First Clinical Department of Cardiology and Electrotherapy, Świętokrzyskie Cardiology Center, 45 Grunwaldzka St, 25-736 Kielce, Poland, phone: +48 697077 060, e-mail: repikus@poczta.onet.pl

Received: 27.01.2014, accepted: 11.04.2014. 


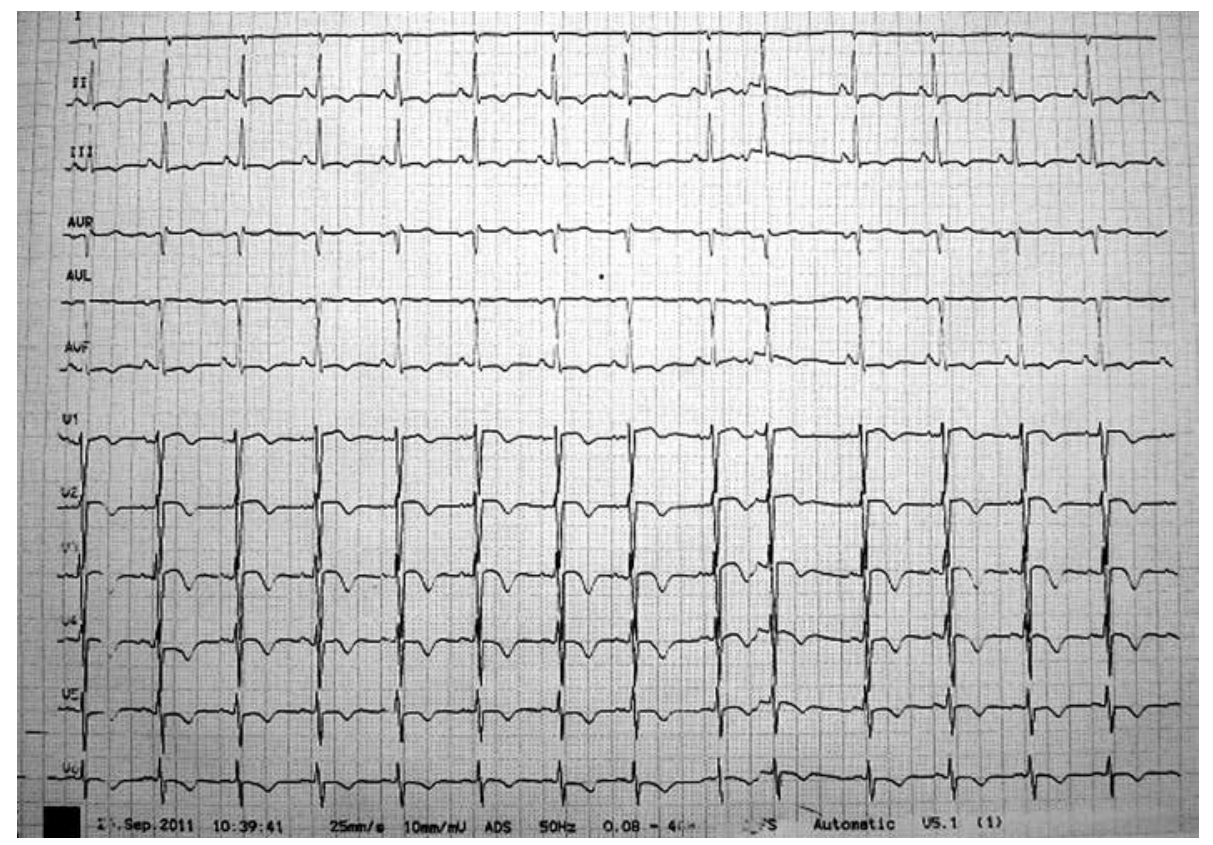

Figure 1. Electrocardiogram. Sinus rhythm 96 per minute. ST segment elevation in leads V1-V3 with positive-negative $T$ waves in these leads. ST suppression up to $1 \mathrm{~mm}$ in II, III, aVF with negative T waves in these leads. Negative T waves in leads V4-V6

about $40 \%$. Increase in the concentration of troponin $\mathrm{T}$ to a maximum value of $0.21 \mathrm{ng} / \mathrm{ml}$ (norm: $0.00-0.03 \mathrm{ng} / \mathrm{ml}$ ) was recorded in the biochemical examination, with the levels of creatine phosphokinase (CPK), creatine kinase $M B(C K-M B)$ and potassium remaining in normal correct ranges. Computed tomography (CT) excluded bleeding to the central nervous system. Cardiac arrest due to ventricular fibrillation in the course of acute coronary syndrome was diagnosed, and urgent coronary angiography was performed, with no atherosclerotic plaques being found in this examination. Acetylsalicylic acid, clopidogrel, low molecular mass heparin, $\beta$-adrenergic antagonist, angiotensin-converting enzyme inhibitor and statin were applied. The patient recovered 14 days after the sudden cardiac arrest, with full respiratory efficiency returning after 21 days of hospitalization. Complete withdrawal of left ventricular contractility disturbances and return of normal systolic left ventricular function were observed in echocardiography. The level of troponin T concentration returned to the normal range. Anginal chest pain did not occur during the remaining time of hospitalization, with the patient's hemodynamic condition being stable. Because of the history of supraventricular tachycardia and having undergone ventricular fibrillation, an electrophysiological study and radiofrequency ablation of focal paroxysmal atrial tachycardia substratum were performed. Ventricular arrhythmia was not released during the programmed ventricle stimulation. The patient was discharged after five weeks' hospitalization to be treated with acetylsalicylic acid $75 \mathrm{mg}$, clopidogrel $75 \mathrm{mg}$ for 12 months, bisoprolol $5 \mathrm{mg}$ per day, ramipril $2.5 \mathrm{mg}$ per day.

\section{Discussion}

Typical features of acute coronary syndrome dominate in the clinical presentation in the initial phase of takotsubo syndrome. The presented patient was admitted to hospital after sudden cardiac arrest with ventricular fibrillation, with ECG showing changes characteristic of myocardial ischemia. The laboratory examination showed a mild increase in the concentration of troponin $\mathrm{T}$, while extensive left ventricle contractility disorders were noted in echocardiography, disproportionately extensive in relation to the slight growth in myocardial necrosis markers. Angiography examination, however, showed no coronary artery stenosis. The described case of acute coronary syndrome met the Mayo Clinic criteria of takotsubo syndrome. The Mayo Clinic criteria for takotsubo are as follows: transient akinesis or dyskinesis of the left ventricular apical and mid-ventricular segments with regional wall-motion abnormalities extending beyond a single epicardial vascular distribution, absence of an obstructive coronary disease or angiographic evidence of acute plaque rupture, new ECG abnormalities: ST-segment elevation or T-wave inversion, absence of recent significant head trauma, intracranial bleeding, pheochromocytoma, hypertrophic cardiomyopathy and myocarditis [2]. In the Polish literature, a large cohort of takotsubo cardiomyopathy (31 patients) was published by Opolski et al., with women constituting almost $93.5 \%$ of patients in the cited study [3]. Takotsubo syndrome etiology remains unclear as yet. Reasons for the disorders have been observed during transient simultaneous 
spasms of all coronary arteries or microvascular dysfunction as a consequence of the overflow effect of catecholamines [4-7]. Some attempts were made at correlating the specific course of the left anterior descending artery (LAD) along the apical diaphragmatic surface of the left ventricle [8].

Sudden cardiac arrest was the first manifestation of takotsubo cardiomyopathy in the presented woman. Associated with catecholaminemia, emotional stress was a triggering factor of the disease. Intensified activation of the sympathetic nervous system leads to increased myocardial cell automatism and intensification of the intracellular calcium cations flow [9]. The increased intracellular calcium level may mediate cardiac arrhythmias [10]. Most takotsubo patients recover in a few days or weeks. In general, the long-term prognosis is good. However, the acute stage of the disease, when strong adrenergic stimulation is activated and myocardial ischemia has arisen, may lead to life-threatening ventricular arrhythmias and critical hemodynamic disorders. The in-hospital average mortality rate from takotsubo cardiomyopathy is $2.5 \%$, being associated with complications such as cardiogenic shock, ventricular arrhythmias (for example torsade de pointes (TdP), ventricular fibrillation), left ventricle rupture, thrombotic and embolic events caused by left ventricle parietal clot, or multiorgan insufficiency [11].

Sudden cardiac death may be the first manifestation of takotsubo cardiomyopathy [12]. Syed et al. on the basis of current medical literature, estimated the frequencies of occurrence of different arrhythmias in takotsubo cardiomyopathy as follows: a prevalence of $2.2 \%$ of ventricular fibrillation, $1.2 \%$ of sustained ventricular tachycardia, $1 \%$ of non-sustained ventricular tachycardia, $0.5 \%$ of asystole, $2.9 \%$ of atrioventricular block, $1.3 \%$ of sinus node dysfunction, $4.7 \%$ of atrial fibrillation, and $1.1 \%$ of sudden cardiac death. These authors also reported that ventricular arrhythmias inducing out-hospital sudden cardiac arrest occurred in $1.1 \%$ of takotsubo cases [11]. Ventricular fibrillation (3.2\%) and sustained ventricular tachycardia (6.4\%) were observed as the most severe forms of arrhythmia in Opolski's study [3]. Moreover, the group with malignant arrhythmia showed significantly wider variation in $R-R$ intervals (maximum $R-R$ interval - minimum $\mathrm{R}-\mathrm{R}$ interval) in the first $24 \mathrm{~h}$ of admission to the hospital [13]. What has also been recorded is an increase in the dispersion of QT, i.e. the difference between the maximum and the minimum QT intervals in a 12-lead ECG, which beside QT and QTc prolongation has been identified as an independent risk factor for sudden death [14-16]. It has been proved that the occurrence of TdP tachycardia in the acute phase of takotsubo cardiomyopathy is associated with QT prolongation, and a cut-off of QTc $>500$ ms has a sensitivity of $82 \%$ and a specificity of $85 \%$ [17]. Additionally, patients with takotsubo cardiomyopathy and TdP had known risk factors for TdP, including bradycardia, hypokalemia, recent conversion from atrial fibrillation to sinus rhythm, use of QT prolonging drugs, and congenital long QT syndrome [18]. It is believed that takotsubo cardiomyopathy is one of the causes of the acquired long QT syndrome and can be a trigger to be able to make overt silent or inapparent congenital long QT syndrome; therefore, all factors that can exacerbate the QT prolongation, i.e. electrolyte imbalances (hypokalemia, hypomagnesemia, hypocalcemia) and use of drugs that would prolong the QT interval, must be removed to prevent an onset of arrhythmia [19]. An implantable cardioverter defibrillator (ICD) implantation should be considered after an episode of sudden cardiac arrest due to ventricular fibrillation in case of takotsubo cardiomyopathy.

Following the recommendations for secondary prevention of sudden cardiac death, implantation of ICD is indicated after an episode of sudden cardiac arrest from ventricular fibrillation, if factors responsible for ventricular fibrillation (VF) are potentially not removable. The VF or sustained VT episodes which occur within $48 \mathrm{~h}$ of myocardial ischemia, considering the reversible cause, are not predictors of other incidents and they are not indications for ICD implantation [20]. A positive result of the programmed ventricular stimulation during an electrophysiology study has been recommended for qualification for ICD implantation. In the presented patient, because of endured sudden cardiac arrest and history of supraventricular arrhythmias, an electrophysiological study was performed and ventricular arrhythmia was not provoked. Some authors suggest that patients with takotsubo cardiomyopathy with risk factors for long QT syndrome, such as QTc > $500 \mathrm{~ms}$, prior syncope, and previous cardiac arrest, may be considered for implantation of an ICD. However, there is no reference in current recommendations to treat patients using electrotherapy. Use of a wearable cardioverter-defibrillator in the acute phase of takotsubo cardiomyopathy, while the left ventricular ejection fraction is under $35 \%$, is one therapeutic option [21].

Prevention of the recurrence of takotsubo cardiomyopathy is discussed in the literature widely. The predominant role of $\beta$-adrenergic drugs is suggested in the treatment of takotsubo disease to suppress harmful effects of the overflow of catecholamines, but reduced recurrence of the disease was not observed. Experimental studies carried out on animals have shown that $\beta$-blockers prevent the recurrence of takotsubo cardiomyopathy and play a protective role in dobutamine-induced stress situation. However, the usage of $\beta$-blockade did not fully prevent the recurrence of apical ballooning syndrome, and also all-cause mortality was higher than in the age - and sex-matched general population [22].

There is no clear guidance on pharmacological treatment after a past takotsubo episode in humans. There 
is a discrepancy as to the use of $\beta$-blockers and double antiplatelet therapy. Fazio et al. revealed the limited usefulness of long-term treatment with $\beta$-blockers [23]. Because of lack of atherosclerotic plaques in coronary angiography, double antiplatelet therapy (acetylsalicylic acid and thienopyridines) for a period of 12 months may be questionable. The assumption is that takotsubo syndrome is an early abortive stage of ST elevation myocardial infarction with spontaneous reperfusion [24]. Different forms of instable atherosclerotic plaques have been shown in intracoronary ultrasonography with the virtual histology option, and erosive plaques constitute $50 \%$ of all cases [25]. In this situation the use of double antiplatelet therapy together with statin could be justifiable.

\section{Conclusions}

Takotsubo cardiomyopathy has not been entirely explained as an etiological disease. As a result, the treatment of this disease is not specific. Even though there is an increasing number of new observations of the course of the disease and more invasive procedures have been performed, there are no homogeneous recommendations of pharmacological treatment and prevention of subsequent episodes of takotsubo cardiomyopathy and arrhythmic events.

\section{References}

1. Budaj A, Beręsewicz A, Undas A. Ischaemic heart disease. In: Internal diseases [Polish]. Szczeklik A (ed). Medycyna Praktyczna, Krakow 2010.

2. Prasad A, Lerman A, Rihal CS. Apical ballooning syndrome (Tako-tsubo or stress cardiomyopathy): mimic of acute myocardial infarction. Am Heart J 2008; 155: 408-17.

3. Opolski G, Pawlak M, Roik M, et al. Clinical presentation, treatment, and long-term outcomes in patients with takotsubo cardiomyopathy. Experience of a single cardiology center. Pol Arch Med Wewn 2010; 120: 231-5.

4. Kurisu S, Sato M, Kawagoe T, et al. Takotsubo-like left ventricular disfunction with ST-segment elevation: a novel cardiac syndrome mimicking acute myocardial infarction. Am Heart J 2002; 143: 448-55.

5. Wittstein IS, Thiemann DR, Lima JAC, et al. Neurohumoral features of myocardial stunning due to sudden emotional stress. N Engl J Med 2005; 352: 539-48.

6. Rotondi F, Manganelli F, Di Lorenzo E, et al. Takotsubo cardiomyopathy in a patient with pacemaker syndrome. Europace 2009; 11: 1712-4.

7. Abraham J, Mudd JO, Kapur NK, et al. Stress cardiomyopathy after intravenous administration of catecholamines and beta-receptor agonists. J Am Coll Cardiol 2009; 53: 1320-5.

8. Ibanez B, Navarro F, Farre J, et al. Takotsubo syndrome associated with a long course of the left anterior descending coronary artery along apical diaphragmic surface of the left ventricle. Rev Esp Cardiol 2004; 57: 209-16.

9. Nef HM, Möllmann H, Akashi YJ, et al. Mechanisms of stress (Takotsubo) cardiomyopathy. Nat Rev Cardiol 2010; 7: 187-93.
10. Thandroyen FT, Morris AC, Hagler HK, et al. Intracellular calcium transients and arrhythmia in isolated heart cells. Circ Res 1991; 69: 810-9.

11. Syed FF, Asirvatham SJ, Francis J. Arrhythmia occurrence with takotsubo cardiomyopathy: a literature review. Europace 2011; 13: 780-8.

12. Akashi YJ, Goldstein DS, Barbaro G, et al. Takotsubo cardiomyopathy: a new form of acute, reversible heart failure. Circulation 2008; 118: 2754-62.

13. Dib C, Prasad A, Friedman PA, et al. Malignant arrhythmia in apical ballooning syndrome: risk factors and outcomes. Indian Pacing Electrophysiol J 2008; 8: 182-92.

14. Rotondi F, Manganelli F, Lanzillo T, et al. Tako-tsubo cardiomyopathy complicated by recurrent torsade de pointes in a patient with anorexia nervosa. Intern Med 2010; 49: 1133-7.

15. Zabel M, Portnoy S, Franz MR. Electrocardiographic indexes of dispersion of ventricular repolarization: an isolated heart validation study. J Am Coll Cardiol 1995; 25: 746-52.

16. Algra A, Tussen JG, Roelandt JR, et al. QTc prolongation measured by standard 12-lead electrocardiography is an independent risk factor for sudden death due to cardiac arrest. Circulation 1991; 83: 1888-94.

17. Behr ER, Mahida S. Takotsubo cardiomyopathy and the long-QT syndrome: an insult to repolarization reserve. Europace 2009; 11: 697-700.

18. Samuel-Kinori L, Kinori M, Kogan Y, et al. Takotsubo cardiomyopathy and QT interval prolongation: who are the patients at risk for torsades de pointes? J Electrocardiol 2009; 42: 353-7.

19. Rotondi F, Manganelli F. Takotsubo cardiomyopathy and arrhythmic risk: the dark side of the moon. Eur Rev Med Pharma Sci 2013; 17: 105-11.

20. Zipes DP, Camm AJ, Borggrefe M. ACC/AHA/ESC 2006 guidelines for management of patients with ventricular arrhythmias and the prevention of sudden cardiac death. Europace 2006; 8: 746837.

21. Deeprasertkul P, Opreanu M, Bianco N, et al. National experience with wearable cardioverter-defibrylator use in takotsubo cardiomyopathy. J Am Coll Cardiol 2013; 10: 361-7.

22. Sharkey SW, Windenburg DC, Lesser JR, et al. Natural history and expansive clinical profile of stress (takotsubo) cardiomyopathy. J Am Coll Cardiol 2010; 55: 333-41.

23. Fazio G, Pizzuto C, Barbaro G, et al. Chronic pharmacological treatment in takotsubo cardiomyopathy. Int J Cardiol 2008; 127: 121-3.

24. Kukla P. Takotsubo and myocardial infarction - similary or the same [Polish]? Kardiol Pol 2012; 70: 241.

25. Pawłowski T, Mintz GS, Kulawik T, et al. Virtual histology intravascular ultrasound evaluation of the left anterior descending coronary artery in patients with transient left ventricular ballooning syndrome. Kardiol Pol 2010; 10: 1093-8. 